\title{
Imagerie du panache de photoablation par laser U.V de différents matériaux cibles
}

\author{
B. Angleraud, C. Germain, C. Girault, C. Champeaux, J. Aubreton et A. Catherinot \\ URA 320, "Céramiques Nouvelles et Traitements de Surfaces", Equipe \\ "Laser-Plasma-Matériaux", Faculté des Sciences, 123 rue Albert Thomas, 87060 Limoges \\ cedex, France
}

Résumé : Cet article est consacré à l'observation à l'aide d'une caméra C.C.D intensifiée du panache plasma créé lors de l'ablation d'un matériau cible (nitrure de bore, carbone, alumine, molybdène, supraconducteur de type $\mathrm{YBaCuO}$ ) par un laser à excimères $\mathrm{KrF}$ $(248 \mathrm{~nm})$. Une série de photographies du plasma a été effectuée sous plusieurs pressions d'azote injecté dans l'enceinte, permettant d'observer les différents régimes d'expansion et de comparer la vitesse de propagation du front du panache pour ces divers matériaux.

Le dispositif d'imagerie utilisé se compose d'un objectif Nikkon de focale $105 \mathrm{~mm}$ et de domaine spectral visible (300-800 nm) monté sur un détecteur C.C.D intensifié Princeton Instruments. Chaque image représente le panache plasma à un temps donné après l'impulsion laser, pour une durée d'intensification de la photocathode du détecteur fixée à $10 \mathrm{~ns}$. La variation du délai s'écoulant entre la prise de la photographie et le début de l'impulsion laser (de $50 \mathrm{~ns}$ à $100 \mu \mathrm{s}$ ) permet de suivre l'évolution temporelle de la forme et de l'aspect du plasma en fonction des conditions expérimentales.

Dans cet article, nous nous limiterons à la description qualitative du panache plasma observé lors de l'ablation d'une cible en nitrure de bore par un laser $\mathrm{KrF}$, à une fluence d'environ $12 \mathrm{~J} / \mathrm{cm}^{2}$.

Sous vide $\left(5 \cdot 10^{-3} \mathrm{~Pa}\right)$, la "bulle plasma" commence à croître dans toutes les directions (fig. a : $\Delta t=90 \mathrm{~ns}$ ) avec une zone lumineuse qui se détache progressivement de la cible. Le nuage grossit et commence à "décoller" de la surface vers $\Delta t=200-300 \mathrm{~ns}$, son expansion coincide alors avec une augmentation de sa dimension et une diminution de son intensité lumineuse (fig. $b$ : $\Delta t=730 \mathrm{~ns}$ ), et il finit par ne plus être détecté vers $900 \mathrm{~ns}$.

Le régime d'expansion change notablement lorsqu'on travaille en présence d'une atmosphère azotée. En effet, les images réalisées avec une pression d'azote de $500 \mathrm{~Pa}$ montrent que le panache, dès 50 ns après limpulsion laser, prend la forme d'un croissant qui s'étend principalement dans la direction parallèle à la surface de la cible. La pression ambiante comprime le plasma et empêche sa libre expansion (fig. $\mathrm{c}: \Delta \mathrm{t}=150 \mathrm{~ns}$ ). Vers 1 $\mu s$, le panache rejoint la cible (fig. d). Ce "retour" du plasma vers la cible s'explique par le fait que la vitesse de déplacement du centre de masse du nuage devient petite (à cause de 
la pression ambiante) par rapport à la vitesse d'expansion dans les trois dimensions : la pression exercée par l'atmosphère d'azote empêche le panache de se détendre librement ; une onde de choc se crée au niveau de l'interface front du panache / atmosphère ambiante et repart en direction de la cible, provoquant un confinement du plasma. Le changement de régime de transport de matière (libre- confiné) apparaît donc clairement lorsque la pression dans l'enceinte varie de $10^{-3}$ à $500 \mathrm{~Pa}$.

Nous avons ensuite cherché à déterminer la vitesse de propagation sous vide du front du plasma pour divers matériaux : nitrure de bore, carbone, alumine, molybdène et supraconducteur YBaCuO. Pour celà, nous avons suivi la position temporelle du front de la bulle avec sa distance par rapport à la cible. Les droites obtenues représentent la vitesse d'expansion dans la direction perpendiculaire à la cible. Il apparaît deux domaines de vitesse assez distincts

- pour les matériaux de masse atomique faible ( $C$ et $B N$ ), les vitesses sont comprises entre 50 et $60 \mathrm{~km} / \mathrm{s}$,

- pour les matériaux de masse atomique plus élevée ( $\left.\mathrm{Mo}, \mathrm{Al}_{2} \mathrm{O}_{3}, \mathrm{YBaCuO}\right)$, les vitesses sont comprises entre 25 et $35 \mathrm{~km} / \mathrm{s}$.

Ces résultats sont tout à fait en accord avec les valeurs de vitesse obtenues précédemment sur ces mêmes matériaux à partir de mesures de temps de vol $[1,2]$.

Références

[1] C. Germain, C. Girault, J. Aubreton and A. Catherinot, Applied Surface Science 69 (1993), 359-364.

[2] B. Angleraud, C. Girault, C.Champeaux, J.Aubreton, A. Catherinot, Proceedings of Diamond films' 94, Il Ciocco (Italy), 25-30 Sept 1994.
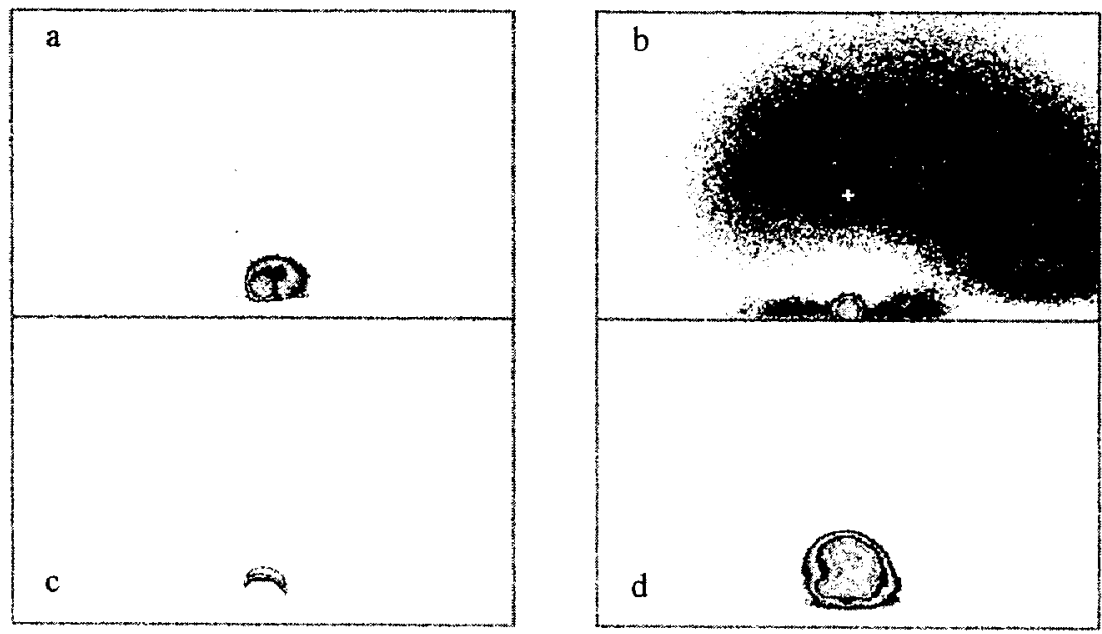